\title{
The Anterior Capsule of the Hip Joint Is a Major Hindrance to the Surgical Treatment of Basicervical Intertrochanteric Fractures: A Single Center, Retrospective Observational Study
}

\author{
Won Chul Shin \\ Pusan National University Yangsan Hospital \\ Jae Hoon Jang \\ Pusan National University Hospital \\ Seok Jin Jung \\ Pusan National University Hospital \\ Nam Hoon Moon ( $\nabla$ namhoonmoon@gmail.com ) \\ Pusan National University Hospital
}

\section{Research Article}

Keywords: hip joint capsule, basicervical intertrochanteric fracture, nonunion, proximal femoral nail antirotation

Posted Date: December 9th, 2020

DOI: https://doi.org/10.21203/rs.3.rs-114007/v1

License: () (1) This work is licensed under a Creative Commons Attribution 4.0 International License. Read Full License 


\section{Abstract}

Background: The basicervical intertrochanteric fracture has recently received considerable attention as one of the major risk factors for failure of internal fixation. We aimed to analyze the anatomical characteristics of basicervical intertrochanteric fractures related to their surgical outcomes using three-dimensional computed tomography (3D CT) and to determine the causes for the high failure rate of surgery.

Methods: Between May 2015 and October 2019, 226 patients with intertrochanteric fracture of the femur including 58 basicervical types who were treated with femoral nail were evaluated. Following data were collected to find out the risk factors for surgical failure: fracture patterns (unstable or basicervical type), tip apex distance, type of postoperative reduction (acceptable or unacceptable), and continuity of anterior capsule for logistic regression analysis to determine the risk factors for surgical failures

Results: Union was achieved in 215 patients (95.1\%), with 11 surgical failures (4.9\%), including 10 non-unions (4.6\%) and 1 osteonecrosis of the femoral head $(0.4 \%)$. Seven surgical failures $(8.7 \%)$ were identified in the unstable fracture group, $6(10.3 \%)$ in the basicervical type group, and $10(19.6 \%)$ in the unacceptable reduction group. Multivariate analysis showed that unstable fracture (OR 10.311, 95\% Cl 1.165 - 91.229, p = 0.036), basicervical type (OR 11.564, $95 \% \mathrm{Cl} 1.339$ - 99.872, $p=0.026$ ), and unacceptable reduction (OR 28.364, 95\% Cl $3.428-234.695, p=0.002$ ) were significant predictors for surgical failure. Unacceptable reduction (OR 13.180, 95\% Cl $1.336-130.059, \mathrm{p}=0.027$ ) was the only risk factor for surgical failure in the basicervical intertrochanteric fracture group. A chi-square test showed that an intact anterior capsule was statistically correlated with surgical failure $(p=0.043)$ and unacceptable reduction $(p=0.008)$.

Conclusion: Because the intact anterior capsule of the hip joint may hinder achieving acceptable reduction, the evaluation of the anterior capsule should be included as an important preoperative assessment for the surgical treatment of basicervical intertrochanteric fracture.

Level of Evidence: Level III, retrospective cohort study

\section{Introduction}

Despite many efforts for the biomechanical understanding of proximal femoral fractures and newly designed intramedullary fixation devices, orthopedic surgeons still experience may failures after internal fixation in elderly patients with intertrochanteric fracture [1-5]. Specifically, the basicervical type of intertrochanteric fracture has recently received considerable attention as one of the major risk factors for failure of internal fixation [6-9]. Although osteosynthesis through internal fixation is the standard surgical treatment for these fractures, studies have emphasized taking special precautions with respect to the high rate of surgical failure. Watson et al. [9] reported 6 failures from 11 patients with twopart basicervical fractures treated with cephalomedullary nailing, even though two of them showed anatomical reduction postoperatively. Based on these results, they asserted the chance of failure in two-part basicervical fractures was high

Nonetheless, the specific factors that contribute to the high failure rate of surgeries for basicervical intertrochanteric fractures have not yet been elucidated. The relationship between fixation device and surgical outcomes has been suggested, but all surgical failures cannot be attributed to implant problems $[6,10,11]$. Several studies have indicated the inherent instability of basicervical intertrochanteric fractures, which is related to their anatomical characteristics, as the cause of the high rate of surgical failure; however, these studies have failed to provide specific statistical data $[9,10,12$, 13].

Thus, in this study, we aimed to analyze the anatomical characteristics of basicervical intertrochanteric fractures related to their surgical outcomes using three-dimensional computed tomography (3D CT) and to determine the causes for the high failure rate of surgery. We hypothesized that failure of anteromedial cortical buttressing would be a risk factor for 
surgical failure of basicervical intertrochanteric fractures and that the anterior capsule of the hip joint could be a main cause of the inherent instability of these fractures.

\section{Materials And Methods}

\section{Research ethics and study population}

This single center observational study was conducted at a tertiary university hospital based on a retrospective review of prospectively collected clinical and radiologic data. The study was conducted in accordance with the tenets of the Declaration of Helsinki. The study protocol was approved by the Pusan National University Hospital Institutional Review Board (H-2008-013-094). Informed consent was obtained from all subjects.

Between May 2015 and October 2019, 348 patients with intertrochanteric fracture of the femur were identified in our institution. Inclusion criteria of this study were patients with intertrochanteric fractures treated with an intramedullary nail. Exclusion criteria included patients who had 1) follow-up time of less than 6 months, 2) pathologic fracture, 3) open fracture, 4) surgery using extra-medullary device, 5) age younger than 65 years, and 6) multiple fractures in the affected limb. A total of 323 patients underwent surgical treatment, and 97 of them were excluded in this study (Fig. 1).

\section{Surgical Principle}

All surgeries were performed on a radiolucent fracture table for fluoroscopic guidance with the patient in the supine position. An intramedullary device (proximal femoral nail anti-rotation and trochanteric femoral nail anti-rotation, Depuy Synthes $\mathrm{GmbH}$, Oberdorf, Switzerland) was used for fracture fixation. The neck shaft angle of the proximal femur on the coronal plane was restored using a traction device on the fracture table in reference to the normal side of the femur, and anteromedial cortical reduction was assessed through a trans-axial image. When posterior displacement of the proximal fragment (unacceptable reduction) was identified on the trans-axial image, indirect reduction was conducted using reduction tools to make a cortical buttress in the anteromedial part of the fracture (Fig. 2). We used a helical blade in all patients and made a tip apex distance of less than $25 \mathrm{~mm}$, and Cleveland index five or eight $[14,15]$.

\section{Radiologic Assessments}

We classified an intertrochanteric fracture as stable or unstable based on Evans' classification [16]. An unstable fracture is an intertrochanteric fracture with a large posteromedial defect. A basicervical intertrochanteric fracture refers to more than $50 \%$ of identified fracture line proximal to the intertrochanteric crest where the anterior capsule of the hip joint is attached to. However, when all fracture lines were located more than $5 \mathrm{~mm}$ proximal to the intertrochanteric crest, the fracture was considered to be a femoral neck fracture. This concept is a modified definition of the basicervical intertrochanteric fracture by Hu et al. [17], and this type of fracture can be either a partial or total an intracapsular fracture (Fig. 3).

Postoperative reduction was divided into three categories, extramedullary, anatomical, and intramedullary reductions based on the displacement of the anteromedial cortex of the proximal fragment on the trans-axial image [2] (Fig. 4). Extramedullary and anatomical reductions were considered acceptable reductions and intramedullary reduction was considered an unacceptable reduction. We assessed the status of the anterior joint capsule using 3D CT in all patients with basicervical intertrochanteric fractures. When the continuity of the anterior capsule was identified without detachment, tear, or avulsion from bony insertion, we considered it to be an intact anterior capsule. When this intact anterior capsule was located between the two fragments, we considered it to be an incarcerated anterior capsule (Fig. 5). 
Radiologic follow-up was conducted $1,3,6,9$, and 12 months after surgery. We defined union as when the cortical bridging was identified at least three cortices in the anteroposterior and lateral image of the hip, with a patient that could ambulate without pain [18]. Non-union was defined as when the proximal or lateral migration of the helical blade, cut-out or cut-through of the helical blade, varus deformation of the proximal fragment, implant failure around the fracture site, and obvious fracture line with antalgic gait at 9 months follow-up X-ray or CT were identified [2]. All radiologic assessments were independently conducted by two orthopedic surgeons who had more than 10 years of experience and had never participated in patient treatment during this study. When they could not reach a consensus, the decision was based on a third orthopedic surgeon's assessment.

\section{Statistical analysis}

Data were analyzed using SPSS software (ver. 17.0 for Windows; IBM, Armonk, NY). Preoperative data including age, sex, bone mass density (BMD), body mass index (BMI), and pre-injury mobility score of enrolled patients were collected based on the review of medical records. Preoperative data were used as covariates with pre- and postoperative radiologic values, including fracture patterns (unstable or basicervical type), tip apex distance, type of postoperative reduction (acceptable or unacceptable), and continuity of anterior capsule for logistic regression analysis to determine the risk factors for surgical failures. After unadjusted analysis, significant variables ( $p$ value of 0.10 ) were included in the model for adjusted analysis. A multivariate logistic regression analysis was performed to examine the association between possible risk factors and surgical failure. We report odds ratios (ORs) and 95\% confidence intervals (Cls) for all associations. A p value of $<0.05$ was considered significant. The inter-observer reliability of all radiologic values was evaluated using the interclass correlation coefficient. The results were interpreted as follows: $>0.8=$ almost perfect agreement, $0.7-0.8=$ strong agreement, $0.5-0.6=$ moderate agreement, $0.3-0.4=$ fair agreement, and $0-0.2=$ poor agreement. $\mathrm{P}<0.05$ was considered statistically significant. Inter-observer reliabilities for unstable fracture, basicervical fracture, postoperative reduction, and continuity of anterior capsule are shown in Table 1. A chi-square test was used to evaluate statistical correlation between continuity of anterior capsule and radiologic values in patients with a basicervical intertrochanteric fracture.

Table 1

Inter-observer reliability of each radiologic values.

\begin{tabular}{|lllll|}
\hline Radiologic values & ICC & $95 \% \mathbf{C l}$ & p-value & Interpretation \\
\hline Unstable fracture & 0.959 & $0.935-0.976$ & $<0.001$ & Almost perfect agreement \\
\hline Basicervical type & 0.872 & $0.834-0.902$ & $<0.001$ & Almost perfect agreement \\
\hline Postoperative reduction & 0.944 & $0.928-0.957$ & $<0.001$ & Almost perfect agreement \\
\hline Intact anterior capsule & 0.741 & $0.562-0.846$ & $<0.001$ & Strong \\
\hline ICC; Inter-observer correlation coefficient, Cl; confident interval & \\
\hline
\end{tabular}

\section{Results}

A total of 226 patients with intertrochanteric fracture were enrolled in the present study. The study participants were 82 male (36.3\%) and 144 female (63.7\%) patients with a mean age of 78.7 (range, 65-99) years and mean follow-up period of 10.0 months. A total of 140 stable (61.9\%) and 86 unstable fractures (38.1\%) were recorded among which 58 (25.7\%) fractures were of basicervical type. Acceptable reduction was identified in 175 patients (77.4\%). Union was achieved in 215 patients (95.1\%), with 11 surgical failures (4.9\%), including 10 non-unions (4.6\%) and 1 osteonecrosis of the femoral head $(0.4 \%)$. 
Seven surgical failures (8.7\%) were identified in the unstable fracture group, 6 (10.3\%) in the basicervical type group, and $10(19.6 \%)$ in the unacceptable reduction group. Univariate analysis showed that basicervical fracture $(\mathrm{OR} 3.762,95 \% \mathrm{Cl}$ 1.103-12.833, $\mathrm{p}=0.034$ ) and unacceptable reduction (OR 42.439, 95\% Cl 5.283-340.926, $\mathrm{p}<0.001$ ) were statistically significant. Multivariate analysis showed that unstable fracture (OR 10.311, 95\% Cl 1.165-91.229, $p=0.036$ ), basicervical type (OR 11.564, 95\% Cl 1.339-99.872, p = 0.026), and unacceptable reduction (OR 28.364, 95\% Cl 3.428234.695, $p=0.002$ ) were significant predictors for surgical failure (Table 2).

Table 2

Logistic regression analysis.

\begin{tabular}{|c|c|c|c|c|c|c|}
\hline \multirow[t]{2}{*}{ variables } & \multirow[t]{2}{*}{ No. } & \multirow{2}{*}{$\begin{array}{l}\text { No. of non-union } \\
\text { (\%) }\end{array}$} & \multicolumn{2}{|l|}{ univariate } & \multicolumn{2}{|l|}{ multivariate } \\
\hline & & & odds ratio $(95 \% \mathrm{Cl})$ & $\begin{array}{l}p \\
\text { value }\end{array}$ & odds ratio $(95 \% \mathrm{Cl})$ & $p_{\text {value }}$ \\
\hline age (years) & - & - & $\begin{array}{l}0.964(0.891- \\
1.043)\end{array}$ & 0.365 & - & - \\
\hline sex & 82 & $3(3.7 \%)$ & 1 (reference) & 0.527 & - & - \\
\hline $\begin{array}{l}\text { male } \\
\text { female }\end{array}$ & 144 & $8(5.6 \%)$ & $\begin{array}{l}1.549(0.399- \\
6.009)\end{array}$ & & & \\
\hline BMD (L-spine, T-score) & - & - & $\begin{array}{l}1.072(0.744- \\
1.544)\end{array}$ & 0.709 & - & - \\
\hline BMD (hip, T-score) & - & - & $\begin{array}{l}1.429(0.802- \\
2.546)\end{array}$ & 0.226 & - & - \\
\hline BMI (kg/m2) & - & - & $\begin{array}{l}1.053(0.888- \\
1.250)\end{array}$ & 0.550 & - & - \\
\hline $\begin{array}{l}\text { preoperative mobility } \\
\text { score }\end{array}$ & - & - & $\begin{array}{l}1.064(0.798- \\
1.419)\end{array}$ & 0.672 & - & - \\
\hline fracture type & 140 & $4(2.9 \%)$ & 1 (reference) & 0.086 & 1 (reference) & 0.036 \\
\hline $\begin{array}{l}\text { stable fracture } \\
\text { unstable fracture }\end{array}$ & 86 & $7(8.1 \%)$ & $\begin{array}{l}3.013(0.855- \\
10.614)\end{array}$ & & $\begin{array}{l}10.311(1.165- \\
91.229)\end{array}$ & \\
\hline basicervical type & 168 & $5(3.0 \%)$ & 1 (reference) & 0.034 & 1 (reference) & 0.026 \\
\hline $\begin{array}{l}\text { no basicervical type } \\
\text { basicervical type }\end{array}$ & 58 & $6(10.3 \%)$ & $\begin{array}{l}3.762(1.103- \\
12.833)\end{array}$ & & $\begin{array}{l}11.564(1.339- \\
99.872)\end{array}$ & \\
\hline tip apex distance & - & - & $\begin{array}{l}1.127(0.908- \\
1.397)\end{array}$ & 0.278 & - & - \\
\hline postoperative & 175 & $1(0.6 \%)$ & 1 (reference) & $<$ & 1 (reference) & 0.002 \\
\hline $\begin{array}{l}\text { acceptable reduction } \\
\text { unacceptable } \\
\text { reduction }\end{array}$ & 51 & $10(19.6 \%)$ & $\begin{array}{l}42.439(5.283- \\
340.926)\end{array}$ & & $\begin{array}{l}28.364(3.428- \\
234.695)\end{array}$ & \\
\hline
\end{tabular}

Among 58 basicervical intertrochanteric fractures, 51 (87.9\%) were stable fractures. Unacceptable reduction was identified in 19 patients (32.8\%) and 30 patients (51.7\%) had an intact anterior capsule. Among six surgical failures in the 
basicervical intertrochanteric fracture group, unacceptable reduction was identified in five patients and intact anterior capsule was identified in five patients. Univariate analysis showed that unacceptable reduction (OR $13.571,95 \% \mathrm{Cl}$ 1.455-126.562, $\mathrm{p}=0.022$ ) had a statistical correlation with surgical failure. Multivariate analysis showed that unacceptable reduction ( $\mathrm{OR} 13.180,95 \% \mathrm{Cl} 1.336-130.059, \mathrm{p}=0.027$ ) was the only risk factor for surgical failure in the basicervical intertrochanteric fracture group (Table 3). The details of the 6 patients with basicervical intertrochanteric fracture who showed surgical failure are presented in Table 4.

Table 3

Logistic regression analysis of basicervical fracture.

\begin{tabular}{|c|c|c|c|c|c|c|}
\hline \multirow[t]{2}{*}{ variables } & \multirow[t]{2}{*}{ No. } & \multirow{2}{*}{$\begin{array}{l}\text { No. of failure } \\
(\%)\end{array}$} & \multicolumn{2}{|l|}{ univariate } & \multicolumn{2}{|l|}{ multivariate } \\
\hline & & & odds ratio $(95 \% \mathrm{Cl})$ & $\begin{array}{l}p \\
\text { value }\end{array}$ & odds ratio (95\% Cl) & $\begin{array}{l}p \\
\text { value }\end{array}$ \\
\hline age (years) & - & - & $0.969(0.888-1.058)$ & 0.486 & - & - \\
\hline sex & 23 & $1(4.3 \%)$ & 1 (reference) & 0.251 & - & - \\
\hline $\begin{array}{l}\text { male } \\
\text { female }\end{array}$ & 35 & $5(14.3 \%)$ & $\begin{array}{l}3.667(0.400- \\
33.639)\end{array}$ & & - & - \\
\hline BMD (L-spine, T-score) & - & - & $1.034(0.654-1.639)$ & 0.885 & - & - \\
\hline BMD (hip, T-score) & - & - & $0.849(0.368-1.959)$ & 0.701 & - & - \\
\hline BMI (kg/m2) & - & - & $0.960(0.748-1.232)$ & 0.749 & - & - \\
\hline $\begin{array}{l}\text { preoperative mobility } \\
\text { score }\end{array}$ & - & - & $0.874(0.572-1.337)$ & 0.536 & - & - \\
\hline fracture type & 51 & $4(7.8 \%)$ & 1 (reference) & 0.116 & - & - \\
\hline $\begin{array}{l}\text { stable fracture } \\
\text { unstable fracture }\end{array}$ & 7 & $2(28.6 \%)$ & $\begin{array}{l}4.700(0.681- \\
32.433)\end{array}$ & & - & - \\
\hline postoperative reduction & 39 & $1(2.6 \%)$ & 1 (reference) & 0.022 & 1 (reference) & 0.027 \\
\hline $\begin{array}{l}\text { acceptable reduction } \\
\text { unacceptable reduction }\end{array}$ & 19 & $5(26.3 \%)$ & $\begin{array}{l}13.571(1.455- \\
126.562)\end{array}$ & & $\begin{array}{l}13.180(1.336- \\
130.059)\end{array}$ & \\
\hline anterior capsule & 28 & $1(3.6 \%)$ & 1 (reference) & 0.077 & - & - \\
\hline $\begin{array}{l}\text { no continuity } \\
\text { continuity }\end{array}$ & 30 & $5(16.7 \%)$ & $\begin{array}{l}7.381(0.804- \\
67.778)\end{array}$ & & - & \\
\hline
\end{tabular}


Table 4

Patients detailed of failed basicervical intertrochanteric fractures.

\begin{tabular}{|c|c|c|c|c|c|c|c|c|c|}
\hline $\begin{array}{l}\text { patients } \\
\text { number }\end{array}$ & sex & $\begin{array}{l}\text { age } \\
\text { (years) }\end{array}$ & $\begin{array}{l}\text { AO } \\
\text { type }\end{array}$ & $\begin{array}{l}\text { BMI } \\
(\mathrm{kg} / \mathrm{m} 2)\end{array}$ & $\begin{array}{l}\text { BMD, } \\
\text { T- } \\
\text { score } \\
\text { (L- } \\
\text { spineo } \\
\text { femur) }\end{array}$ & $\begin{array}{l}\text { continuity of } \\
\text { anterior } \\
\text { capsule }\end{array}$ & implant & $\begin{array}{l}\text { postoperative } \\
\text { reduction }\end{array}$ & outcomes \\
\hline 1 & female & 85 & A12 & 20.0 & $\begin{array}{l}-1.4 / \\
-2.4\end{array}$ & + & PFNA & unacceptable & $\begin{array}{l}\text { non-union } \\
\text { with } \\
\text { proximal } \\
\text { migration } \\
\text { of helical } \\
\text { blade }\end{array}$ \\
\hline 2 & female & 76 & A12 & 28.7 & $\begin{array}{l}-1.3 / \\
-2.7\end{array}$ & + & PFNA & unacceptable & $\begin{array}{l}\text { non-union } \\
\text { with } \\
\text { lateral } \\
\text { migration } \\
\text { of helical } \\
\text { blade }\end{array}$ \\
\hline 3 & female & 86 & A12 & 19.4 & $\begin{array}{l}0.7 / \\
-2.2\end{array}$ & - & PFNA & acceptable & $\begin{array}{l}\text { FHON } \\
\text { with } \\
\text { protrusion } \\
\text { of helical } \\
\text { blade }\end{array}$ \\
\hline 4 & female & 78 & A12 & 22.6 & $\begin{array}{l}-3.1 / \\
-2.8\end{array}$ & + & PFNA & unacceptable & $\begin{array}{l}\text { non-union } \\
\text { with } \\
\text { excessive } \\
\text { sliding of } \\
\text { helical } \\
\text { blade }\end{array}$ \\
\hline 5 & female & 74 & A23 & 21.5 & $\begin{array}{l}-3.2 / \\
-2.5\end{array}$ & $\begin{array}{l}+ \\
\text { (incarcerated) }\end{array}$ & TFNA & unacceptable & $\begin{array}{l}\text { non-union } \\
\text { with } \\
\text { proximal } \\
\text { migration } \\
\text { of helical } \\
\text { blade }\end{array}$ \\
\hline 6 & male & 86 & A23 & 18.7 & $\begin{array}{l}-1.0 / \\
-1.9\end{array}$ & $\begin{array}{l}+ \\
\text { (incarcerated) }\end{array}$ & TFNA & unacceptable & $\begin{array}{l}\text { non-union } \\
\text { with } \\
\text { proximal } \\
\text { migration } \\
\text { of helical } \\
\text { blade }\end{array}$ \\
\hline
\end{tabular}

Four out of six of the surgical failures in the basicervical intertrochanteric fracture group presented with A12 fractures, while the two other patients presented with A23 fractures based on the AO classification. When we excluded 11 patients with non-displaced fracture (A11) out of 58 basicervical intertrochanteric fractures, a chi-square test showed that an intact anterior capsule was statistically correlated with surgical failure $(p=0.043)$ and unacceptable reduction $(p=0.008)$ (Table 5). 
Table 5

Comparison of failure and postoperative reduction based on the continuity of anterior capsule in basicervical intertrochanteric fracture except 11 undisplaced fractures (A11).

\begin{tabular}{|c|c|c|c|}
\hline variables & continuity + & continuity - & $p$ value \\
\hline No. & $22(46.8)$ & $25(53.2)$ & - \\
\hline Failures & $5(10.6)$ & $1(2.1)$ & 0.043 \\
\hline \multicolumn{4}{|c|}{ Postoperative reduction } \\
\hline Acceptable & $9(40.9)$ & $20(80.0)$ & \multirow[t]{2}{*}{0.008} \\
\hline Unacceptable & $13(59.1)$ & $5(20.0)$ & \\
\hline Variables are $p$ & the number & & \\
\hline
\end{tabular}

\section{Discussion}

This study defines a basicervical intertrochanteric fracture as when more than $50 \%$ of the fracture line is located proximal to the intertrochanteric crest where the anterior capsule of the hip joint is attached. This study evaluated the incidence and characteristics to determine the factors associated with surgical failures. A total of 58 of 226 patients (25.7\%) with intertrochanteric fracture enrolled in this study showed basicervical intertrochanteric fracture. Among eleven surgical failures, six (10.3\%) were identified in the basicervical intertrochanteric fracture group, and the basicervical type was a significant predictor for surgical failure in all patients with intertrochanteric fractures. Postoperative unacceptable reduction was the only significant predictor for surgical failure in 58 basicervical intertrochanteric fractures and no significant association between an intact anterior capsule and surgical failure was identified. However, five out of six surgical failures, excluding one case of osteonecrosis of the femoral head, showed unacceptable reduction and an intact anterior capsule including two incarcerated capsules. When we excluded 11 patients with non-displaced fractures (A11) out of the 58 patients with basicervical intertrochanteric fractures, an intact anterior capsule was statistically correlated with surgical failure and posterior reduction.

This study showed that a basicervical fracture type is one of the significant risk factors for surgical failure of an intertrochanteric fracture. Previous studies have suggested the high susceptibility of basicervical intertrochanteric fractures to surgical failure. Su et al. [12] suggested that basicervical fractures may have increased biomechanical instability and a higher tendency to collapse compared with other types of intertrochanteric fractures. Bojan et al. [19] reported $9 \%$ of screw cutout rate in patients with basicervical fracture compared with $1 \%$ of screw cutout rate overall in a large series of more than 3,000 fractures in the trochanteric regions. Watson et al. [9] suggested the inherent instability of basicervical proximal femoral fractures makes treatment more difficult based on the surgical result when treated with a cephalomedullary fixation. Kwak et al. [10] mentioned that the inherent instability of basicervical proximal femoral fractures was associated with the anatomical vulnerability of this fracture, including the narrow cortical base of the proximal fragment and the subsequent narrow contact area at the main fracture site, along with insufficient cancellous interdigitation compared with other types of intertrochanteric fractures. Furthermore, the short proximal fragment of this fracture can cause rotational instability during bone healing regardless of fixation devices. For these reasons, achieving anteromedial cortical buttressing around the fracture site of the basicervical intertrochanteric fracture postoperatively may be a vital aspect of surgery. Anteromedial cortical buttressing has been previously emphasized in unstable intertrochanteric fractures $[2,20]$. However, to the best of our knowledge, the importance of anterior medial cortical buttressing in basicervical intertrochanteric fracture has not yet been elucidated. We believe that the biomechanical understanding of the proximal femur from previous studies and the surgical results of our study might offer enough theoretical evidence to support the importance of anteromedial cortical buttressing in these fractures. 
We focused on the fact that 4 out of 6 surgical failures in the basicervical intertrochanteric fracture group were associated with displaced two-part fracture corresponding to A12 fracture based on the AO classification. The incidence of unacceptable reduction in this group was $32.8 \%$ (19 out of 58 ) despite efforts to achieve acceptable reduction under fluoroscopic guidance. In particular, among 35 A12 fractures, 13 patients (37.1\%) showed unacceptable reduction, which was a higher incidence than that shown in all patients with intertrochanteric fractures enrolled in this study (22.6\%). This result indicates that achieving an acceptable reduction in an A12 type of basicervical intertrochanteric fracture can be more difficult than in other types of intertrochanteric fractures.

High incidences of postoperative unacceptable reduction in A12 fractures may be attributed to the radiologic misunderstanding of fluoroscopic trans-axial images during surgery. When there is distraction of fracture site, even though intraoperative reduction seems anatomical, it can become an unacceptable reduction during follow-up. This is because the sliding of the proximal fragment occurs from the anterior to the posterior, along the direction of the helical blade. In this study, four out of five patients with non-union in the basicervical intertrochanteric group showed unacceptable reduction in the follow-up radiographs, even though they showed anatomical reduction on intraoperative fluoroscopy and postoperative radiographs. Due to the distraction of the fracture site regarding excessive traction or intentional valgus reduction during the surgery, the intraoperative fluoroscopic images and postoperative radiographs may be misconstrued as anatomical reduction. The fracture gap can lead to the failure of anteromedial cortical buttressing and intramedullary reduction during follow-up, because the sliding direction of the proximal fragment is consistent with the direction of the helical blade. Thus, slight over-reduction should be considered for the surgical treatment of basicervical intertrochanteric fracture when the distraction of fracture site is expected.

We attempted to prove our hypothesis that the anterior capsule of the hip joint is a potential inherent vulnerability that may make it difficult to achieve acceptable reduction. The anterior capsule of a basicervical intertrochanteric fracture can be intactly attached distally to the fracture site because this fracture shows the characteristics of a partially or totally intra-capsular fracture. If the fracture occurred without detachment of the anterior capsule from its distal insertion, it may be a considerable hindrance to the accurate reduction or proper sliding of the proximal fragment. We identified five cases with an intact anterior capsule out of six surgical failures in the basicervical intertrochanteric fracture group. When we excluded one case with osteonecrosis of the femoral head, all patients with non-union had an intact anterior capsule, and two cases showed incarceration of the anterior capsule in the fracture site. Based on these results, we believe that the anterior capsule of the hip joint can be a major hindrance that negatively affects fracture reduction during surgery.

The present study has several limitations. First, this is a retrospective study with relatively few enrolled cases of surgical failures, which might have affected the reliability of the logistic regression analysis. Second, despite high inter-observer reliability achieved, radiologic evaluation for the anterior capsule using 3D CT has not been previously validated. Furthermore, although the anterior capsule of the hip joint and surgical failure of the basicervical intertrochanteric fracture may be correlated, studies offering clinical evidence on the biomechanical effect of the anterior capsule on the surgical outcome are needed. Third, although we defined basicervical intertrochanteric fractures using a modified concept, this definition might be ambiguous. In addition, although the inter-class correlation coefficient of this fracture was 0.856 in this study, which can be interpreted as almost perfect agreement, identifying the insertion site of the anterior capsule and setting the boundary between the femoral neck and the basicervical intertrochanteric fracture can be subjective. Finally, this study did not suggest a surgical solution to overcome the inherent vulnerability of basicervical intertrochanteric fractures. Based on the results of the present study, anterior capsulectomy might contribute to achieving proper anteromedial cortical buttressing. However, additional studies are need to determine if this surgical technique can improve surgical outcomes or if it may take a toll on the soft tissue around the hip joint with additional blood loss.

\section{Conclusion}


We determined that the basicervical fracture type is a significant predictor for surgical failure in intertrochanteric fractures. Due to its inherent instability, achieving anteromedial buttressing is crucial to improving the stability around the fracture site in basicervical intertrochanteric fractures. Because the intact anterior capsule of the hip joint may hinder achieving acceptable reduction, the evaluation of the anterior capsule should be included as an important preoperative assessment for the surgical treatment of basicervical intertrochanteric fracture. In addition, further studies detailing potential surgical solutions to achieve acceptable reduction in basicervical intertrochanteric fractures with an intact anterior capsule are also needed.

\section{Declarations}

\section{Ethics approval and consent to participate}

The study protocol was approved by the Pusan National University Hospital Institutional Review Board (H-2008-013-094). Informed consent was obtained from all subjects.

\section{Consent for publication}

Not applicable.

\section{Availability of data and materials}

The datasets used and/or analyzed during the current study are available from the corresponding author upon reasonable request.

\section{Competing interests}

The authors declare that they have no financial and non-financial competing interests.

\section{Funding}

No funding was obtained for this study.

\section{Acknowledgement}

This study was supported by Biomedical Research Institute Grant (20200011), Pusan National University Hospital.

\section{Author's contribution}

Won Chul Shin and Nam Hoon Moon were responsible for the study design and writing the manuscript.

Jae Hoon Jang and Seok Jin Jung were responsiable for performing data collection and analysis.

All authors reviewed the manuscript.

\section{References}

1. Moon NH, Shin WC, Kim JS, Woo SH, Son SM, Suh KT. Cementless total hip arthroplasty following failed internal fixation for femoral neck and intertrochanteric fractures: A comparative study with 3-13 years' follow-up of 96 consecutive patients. Injury. 2019;50:713-9.

2. Shin WC, Jang JH, Jeong JY, Suh KT, Moon NH. Effect of a synthetic osteoconductive bone graft substitute with zeta potential control (geneX $((\mathrm{R})) \mathrm{ds}$ ) in the treatment of intertrochanteric fracture: A single center experience of 115 
consecutive proximal femoral nail antirotations. J Orthop Sci. 2019;24:842-9.

3. Socci AR, Casemyr NE, Leslie MP, Baumgaertner MR. Implant options for the treatment of intertrochanteric fractures of the hip: rationale, evidence, and recommendations. Bone Joint J. 2017;99:128-33.

4. Futamura K, Baba T, Homma Y, Mogami A, Kanda A, Obayashi O, et al. New classification focusing on the relationship between the attachment of the iliofemoral ligament and the course of the fracture line for intertrochanteric fractures. Injury. 2016;47:1685-91.

5. Li JT, Han L, Zhang H, Zhao Z, Su XY, Zhou JF, et al. Medial sustainable nail versus proximal femoral nail antirotation in treating AO/OTA 31-A2.3 fractures: Finite element analysis and biomechanical evaluation. Injury. 2019;50:648-56.

6. Johnson J, Deren M, Chambers A, Cassidy D, Koruprolu S, Born C. Biomechanical Analysis of Fixation Devices for Basicervical Femoral Neck Fractures. J Am Acad Orthop Sur. 2019;27:41-8.

7. Xiong WF, Du SC, Chang SM. Choosing an optimal implant fixation for basicervical femoral neck fractures. Injury. 2018;49:1238-9.

8. Lee YK, Yoon BH, Hwang JS, Cha YH, Kim KC, Koo KH. Risk factors of fixation failure in basicervical femoral neck fracture: Which device is optimal for fixation? Injury. 2018;49:691-6.

9. Watson ST, Schaller TM, Tanner SL, Adams JD, Jeray KJ. Outcomes of Low-Energy Basicervical Proximal Femoral Fractures Treated with Cephalomedullary Fixation. J Bone Joint Surg Am. 2016;98:1097-102.

10. Kwak DK, Kim WH, Lee SJ, Rhyu SH, Jang CY, Yoo JH. Biomechanical Comparison of Three Different Intramedullary Nails for Fixation of Unstable Basicervical Intertrochanteric Fractures of the Proximal Femur: Experimental Studies. Biomed Res Int. 2018;11:2018.

11. Okano I, Sawada T, Kushima N, Tachibana T, Inagaki K. Treatment With Helical Blade Cephalomedullary Nail for Two-Part Basicervical Proximal Femoral Fracture in Elderly Patients: A Retrospective Observational Study. Geriatr Orthop Surg. 2017;8:244-51.

12. Su BW, Heyworth BE, Protopsaltis TS, Lipton CB, Sinicropi SM, Chapman CB, et al. Basicervical versus intertrochanteric fractures: an analysis of radiographic and functional outcomes. Orthopedics. 2006;29:919-25.

13. Tan BY, Lau AC, Kwek EB. Morphology and fixation pitfalls of a highly unstable intertrochanteric fracture variant. J Orthop Surg (Hong Kong). 2015;23:142-5.

14. Baumgaertner MR, Solberg BD. Awareness of tip-apex distance reduces failure of fixation of trochanteric fractures of the hip. J Bone Joint Surg Br. 1997;79:969-71.

15. Baumgaertner MR, Curtin SL, Lindskog DM, Keggi JM. The value of the tip-apex distance in predicting failure of fixation of peritrochanteric fractures of the hip. J Bone Joint Surg Am. 1995;77:1058-64.

16. Knobe M, Gradl G, Ladenburger A, Tarkin IS, Pape HC. Unstable intertrochanteric femur fractures: is there a consensus on definition and treatment in Germany? Clin Orthop Relat Res. 2013;471:2831-40.

17. Hu SJ, Yu GR, Zhang SM. Surgical treatment of basicervical intertrochanteric fractures of the proximal femur with cephalomeduallary hip nails. Orthop Surg. 2013;5:124-9.

18. Corrales LA, Morshed S, Bhandari M, Miclau T, 3rd. Variability in the assessment of fracture-healing in orthopaedic trauma studies. J Bone Joint Surg Am. 2008;90:1862-8.

19. Bojan AJ, Beimel C, Taglang G, Collin D, Ekholm C, Jonsson A. Critical factors in cut-out complication after Gamma Nail treatment of proximal femoral fractures. BMC Musculoskelet Disord. 2013;14:1.

20. Kim Y, Dheep K, Lee J, Yoon YC, Shon WY, Oh CW, et al. Hook leverage technique for reduction of intertrochanteric fracture. Injury. 2014;45:1006-10.

\section{Figures}

Page $11 / 16$ 
Between May 2015 and October 2019,

348 patients with intertrochanteric fracture

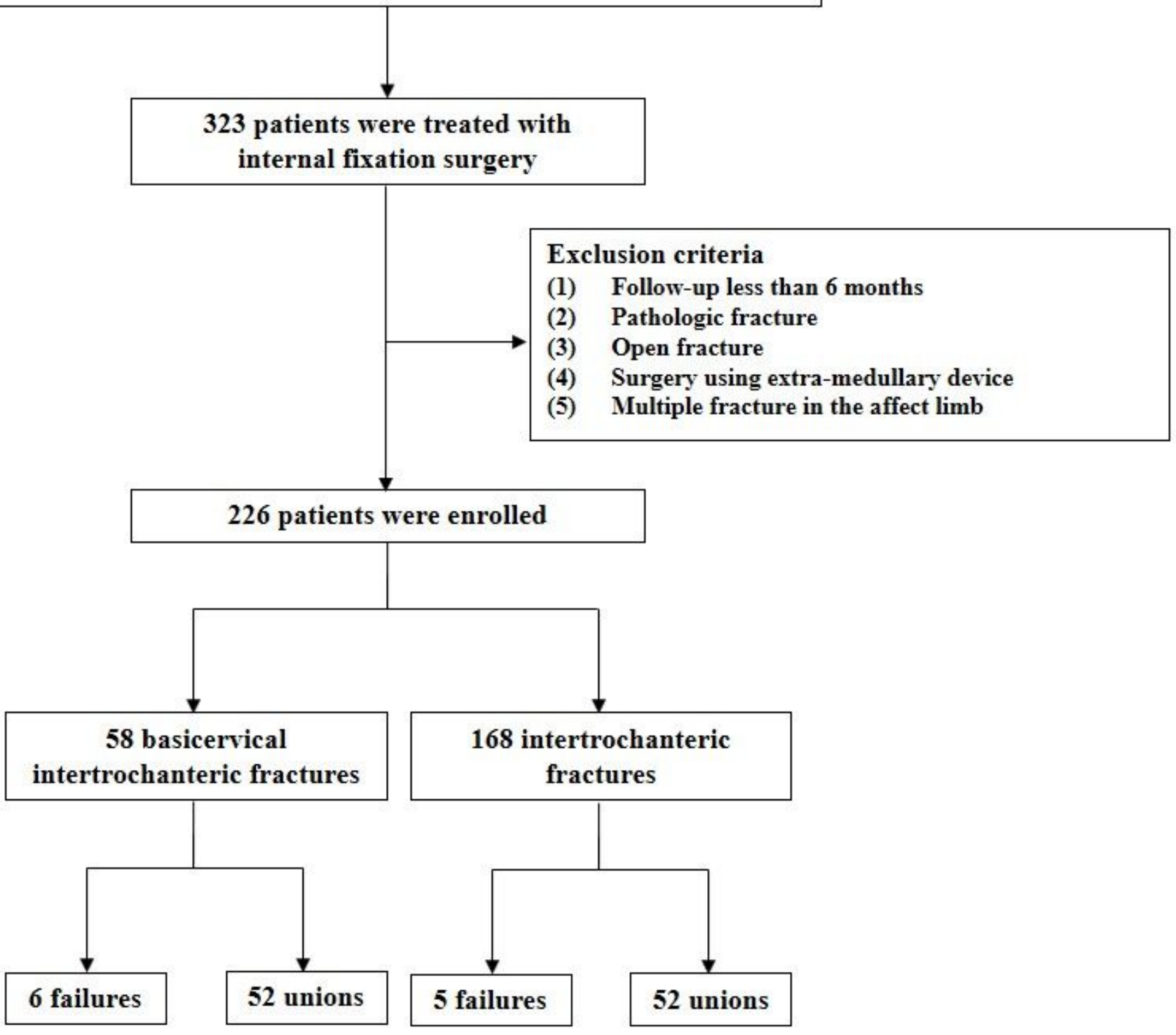

Figure 1

Flow chart of the study. 


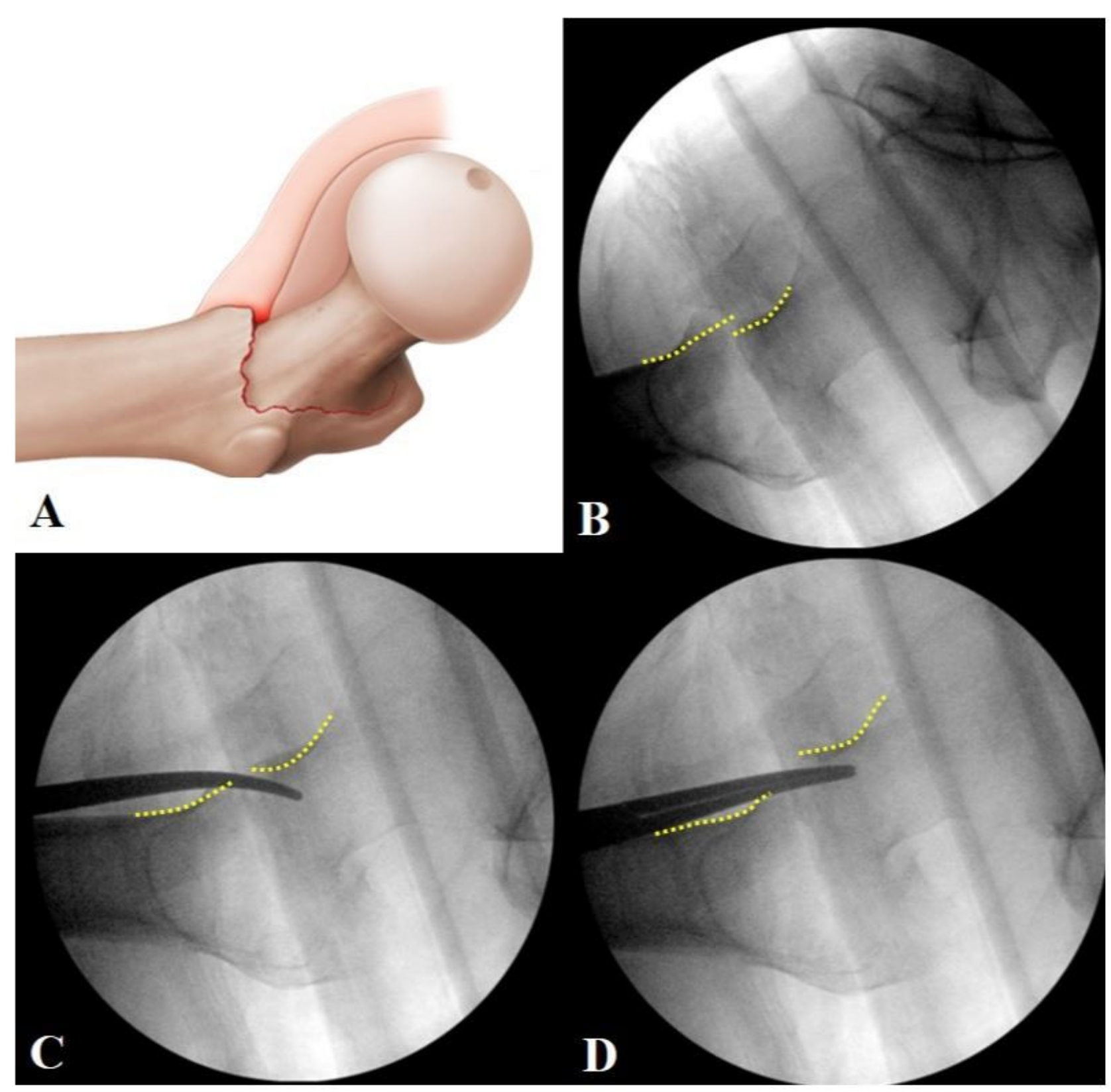

Figure 2

(A) Posterior displacement of the proximal fragment with an intact anterior capsule of the hip joint attached to the distal part of the fracture. (B) Fluoroscopic image showing intramedullary reduction. (C, D) Indirect reduction using reduction tools to make a cortical buttress in the anteromedial part of the fracture. 


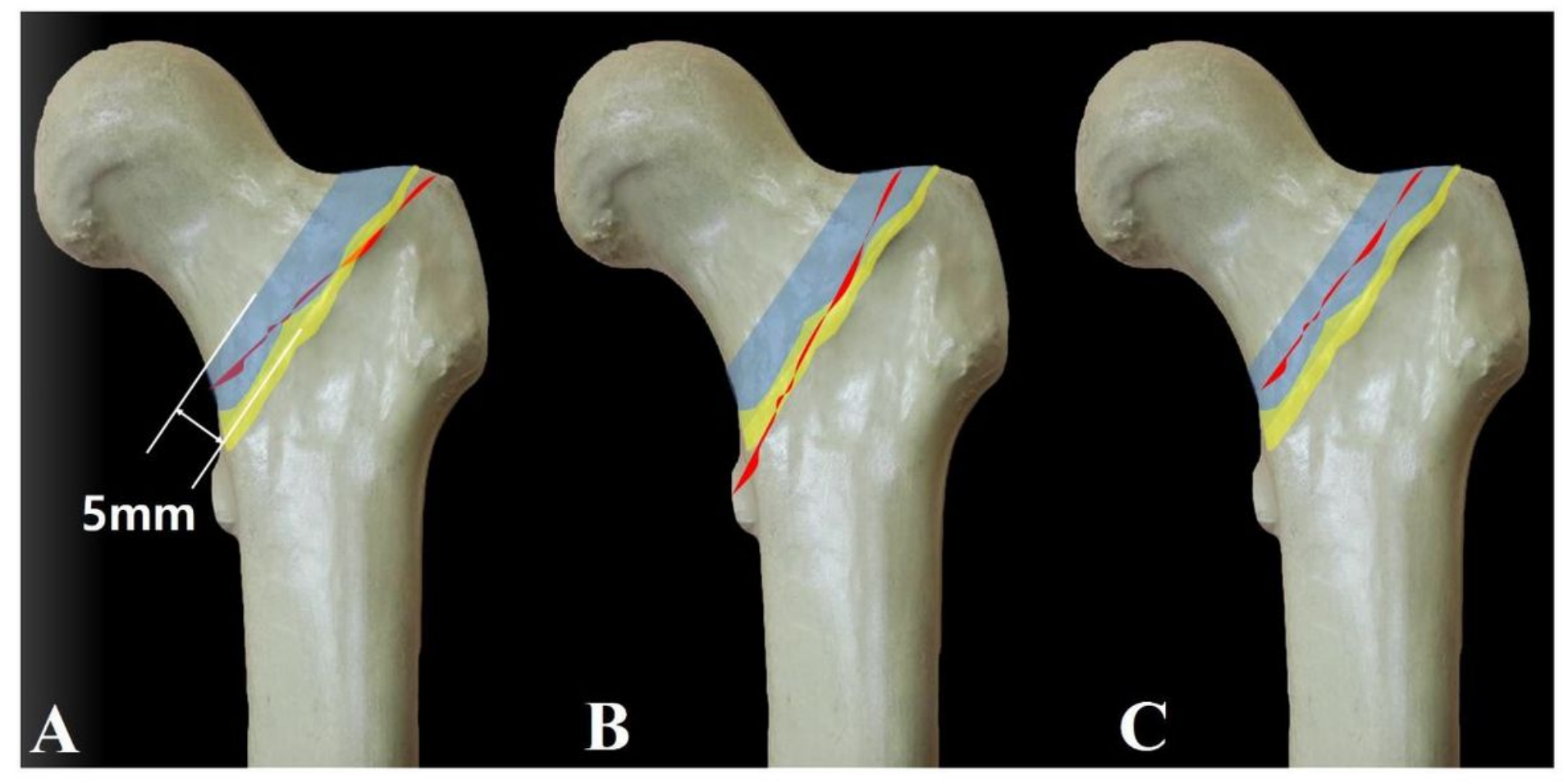

\section{Figure 3}

Three types of basicervical intertrochanteric fractures. (A) Distally intracapsular. (B) Proximally intracapsular, (C) Totally intracapsular. 


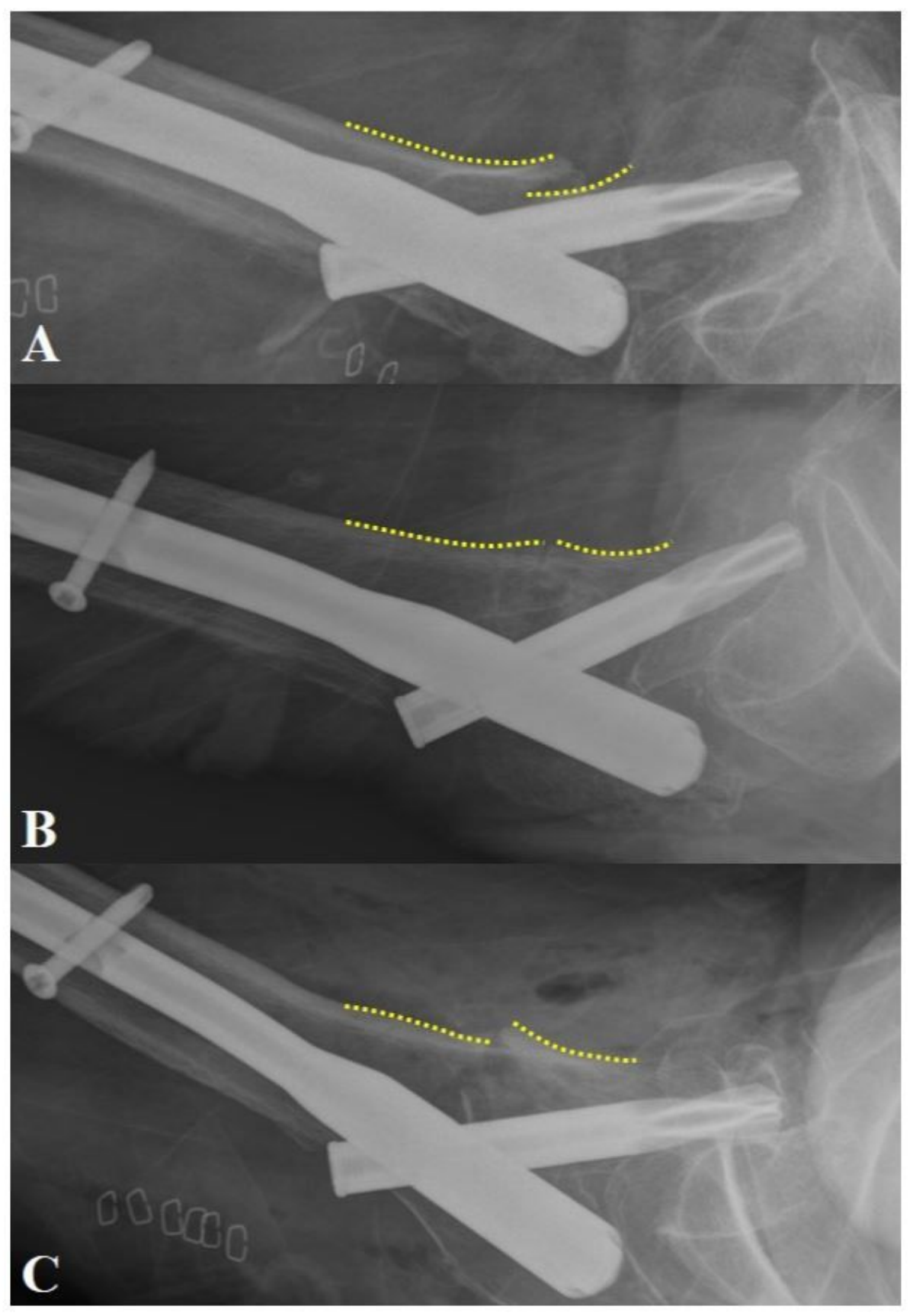

\section{Figure 4}

Classification of postoperative reduction. (A) Intramedullary reduction. (B) Anatomical reduction. (C) Extramedullary reduction. 


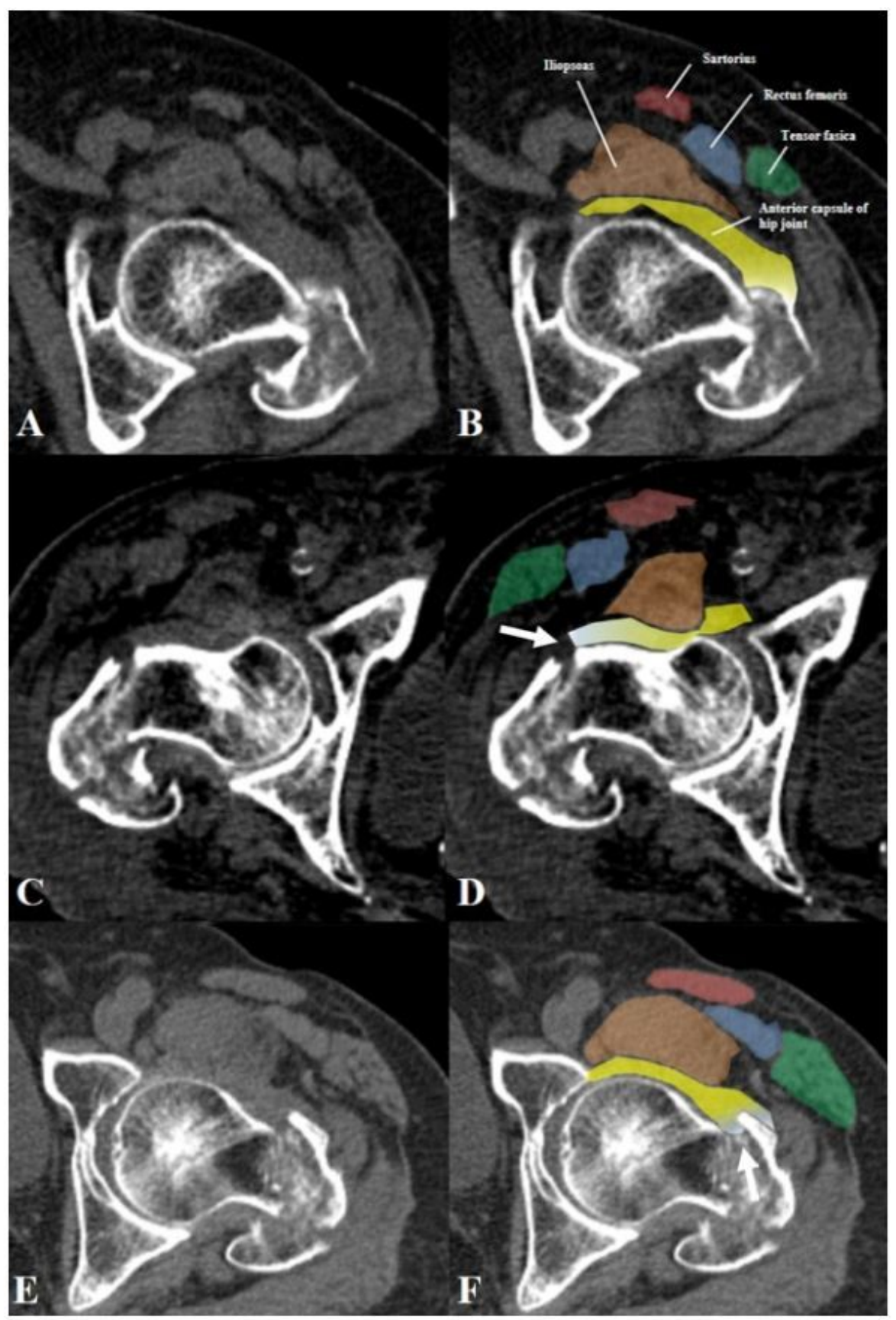

Figure 5

Radiologic evaluation of an anterior capsule of the hip joint. (A, B) Intact. (C, D) Detached. (E, F) Incarcerated. 